Document downloaded from:

http://hdl.handle.net/10251/169954

This paper must be cited as:

Shitomi, T.; Garro, E.; Murayama, K.; Gomez-Barquero, D. (2017). Performance evaluation of MIMO channel estimation for ATSC 3.0. IEEE. 1-5.

https://doi.org/10.1109/BMSB.2017.7986237

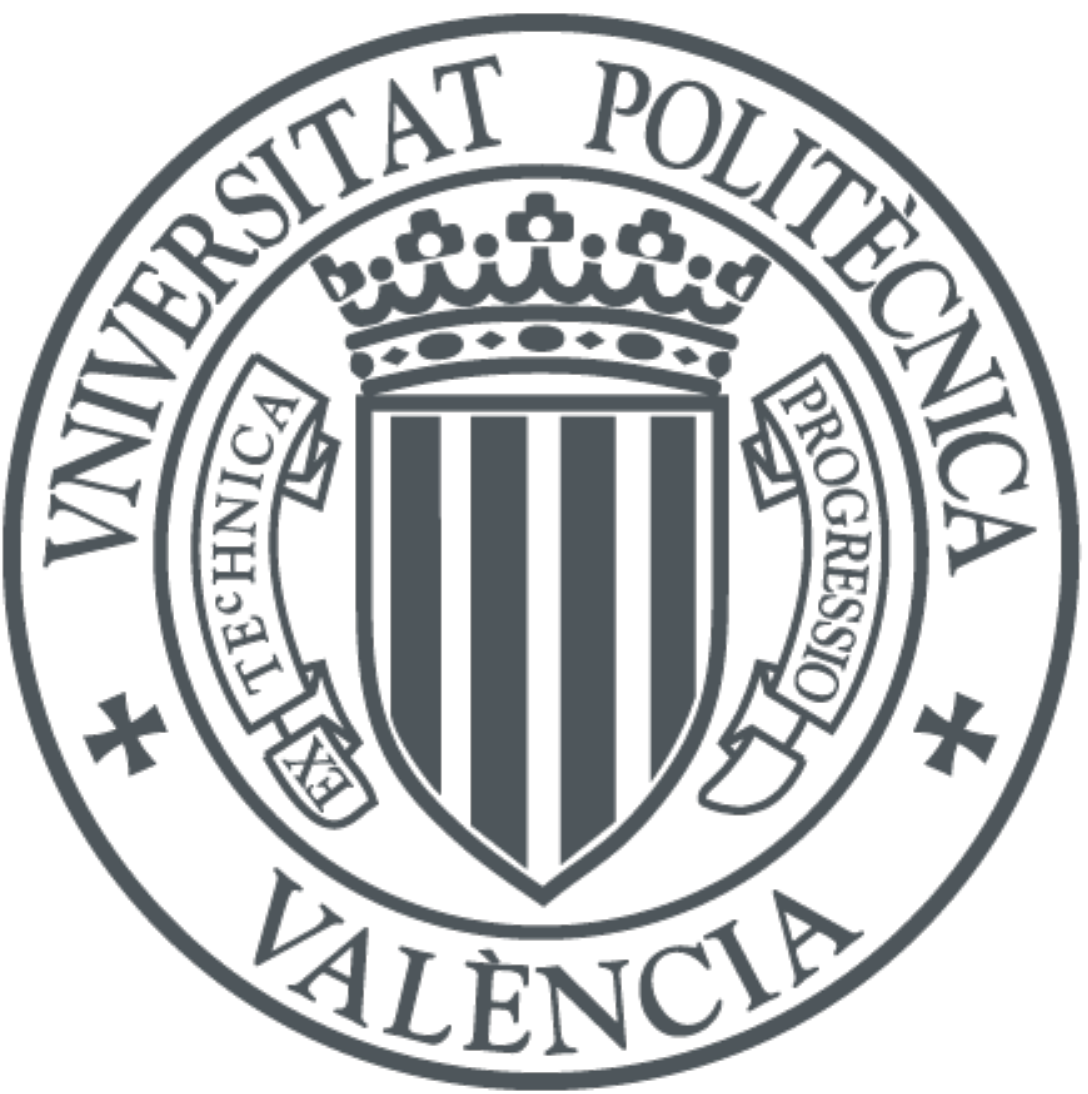

The final publication is available at

https://doi.org/10.1109/BMSB.2017.7986237

Copyright IEEE

Additional Information 


\title{
Performance Evaluation of MIMO Channel Estimation for ATSC 3.0
}

\author{
Takuya Shitomi $^{\dagger \ddagger}$, Eduardo Garro ${ }^{\ddagger}$, Kenichi Murayama ${ }^{\dagger}$ and David Gomez-Barquero ${ }^{\ddagger}$ \\ ${ }^{\dagger}$ Japan Broadcasting Corporation \\ Tokyo, Japan \\ Email: \{shitomi.t-gy, murayama.k-fu\}@nhk.or.jp \\ ¥iTEAM Research Insitute, Universitat Politecnica de Valencia \\ Valencia, Spain \\ Email: \{tashi1, edgarcre, dagobar\}@iteam.upv.es
}

\begin{abstract}
ATSC 3.0, the latest Digital Terrestrial Television (DTT) standard, allows a higher spectral efficiency and/or a transmission robustness with Multiple-Input Multiple-Output (MIMO) technology compared to existing single-antenna DTT networks. Regarding MIMO channel estimation, two pilot encoding algorithms known as Walsh-Hadamard encoding and Null pilot encoding are possible in ATSC 3.0. The two MIMO pilot algorithms are standardized so as to have the same pilot positions and the same pilot boosting as SISO, but the performance has not been evaluated. This paper focuses on the performance evaluation of the two MIMO pilot encoding algorithms in ATSC 3.0 using physical layer simulations. Results can be used as guidelines or recommended practices to broadcasters to select the MIMO pilot encoding algorithm that better suits their service requirments. Several channel estimation algorithms have been evaluated in both mobile and fixed reception conditions. The simulation results show that Null pilot encoding provides slightly better performance than Walsh-Hadamard encoding for fixed reception but worse performance for mobile reception, especially at high signal-tonoise ratios.
\end{abstract}

Keywords-ATSC 3.0; terrestrial broadcasting; MIMO; channel estimation; pilot pattern.

\section{INTRODUCTION}

Multiple-Input Multiple-Output (MIMO) antenna technology was first time introduced in Digital Terrestrial Television (DTT) specification DVB-NGH [1] and it has been further developed and fully standardized in ATSC 3.0 [2], [3]. MIMO technology provides a higher spectral efficiency via spatial multiplexing, and/or a higher transmission robustness via spatial diversity. In practice, MIMO in DTT is implemented using cross-polarized 2x2 MIMO, i.e. horizontal and vertical polarization to decorrelate the channel in Line-of-Sight (LOS) reception conditions [4].

2x2 MIMO spatial multiplexing requires doubling the pilot overhead compared to SISO (Single-Input Single-Output) to keep the channel estimation performance. DVB-NGH adopted an orthogonal scattered pilot encoding scheme, namely WalshHadamard (WH) encoding, which is the same configuration used in DVB-T2 for Multiple-Input Single-Output (MISO) [5]. ATSC 3.0 adopted Walsh-Hadamard encoding for MIMO channel estimation together with another orthogonal scattered pilot encoding algorithm known as Null pilot (NP) encoding [3].

Although different studies have shown the pilot optimization for SISO in ATSC 3.0, see e.g. [6], the impact of pilot encoding for MIMO transmission has not been fully evaluated. Indeed, the two pilot encoding algorithms were not deeply compared in the standardization process based on different channel estimation algorithms and channel conditions [3]. Moreover, the MIMO pilots are directly standardized so as to have the same positions (pilot patterns) and the same amplitudes (pilot boosting) as SISO.

This paper evaluates the MIMO pilot encoding with two kinds of channel interpolator for the receiver. The evaluation is conducted with several pilot boosting in both fixed and mobile reception scenarios. Performance results are extracted from physical layer simulations with an ATSC 3.0 simulator.

\section{ATSC 3.0 MIMO PILOTS}

Two pilot encoding algorithms are possible in ATSC 3.0 for MIMO scattered pilots. MIMO scattered pilots fall on exactly the same positions as for SISO, but the amplitudes and/or phases may be modified compared to SISO. The terminology employed for the MIMO pilot patterns is described as MPa_b, where $\mathrm{a}=D_{\mathrm{X}}$ and $\mathrm{b}=D_{\mathrm{Y}}$ are defined. $D_{\mathrm{X}}$ is the number of carriers between the scattered pilot bearing carriers and $D_{\mathrm{Y}}$ is the number of symbols between the scattered pilots in a single pilot bearing carrier.

Fig. 1 illustrates the MIMO scattered pilot MP3_2, i.e. $D_{\mathrm{X}}$ $=3, D_{\mathrm{Y}}=2$, for Walsh-Hadamard encoding and Null pilot encoding. The differences between the two MIMO pilot encoding algorithms are described next.

\section{A. Walsh-Hadamard}

With Walsh-Hadamard encoding, the phases of all pilots transmitted from antenna \#1 are not modified from SISO. Regarding the signal transmitted from antenna \#2, the pilots are partitioned into two subsets, and the phases of the scattered pilots are inverted every second pilot bearing carrier. That results that the half of scattered pilots transmitted from antenna \#2 are not modified and that the other half scattered pilots are inverted. As the result of the phase inversion on every second pilot bearing carrier, the number of carriers between the scattered pilot bearing carriers in each subset is doubled. After time interpolation on every scattered pilot bearing carrier, frequency interpolation is performed separately on each subset. Consequently, the Nyquist limit of the channel estimation in frequency falls to half compared to the uncoded scattered pilot in SISO. 

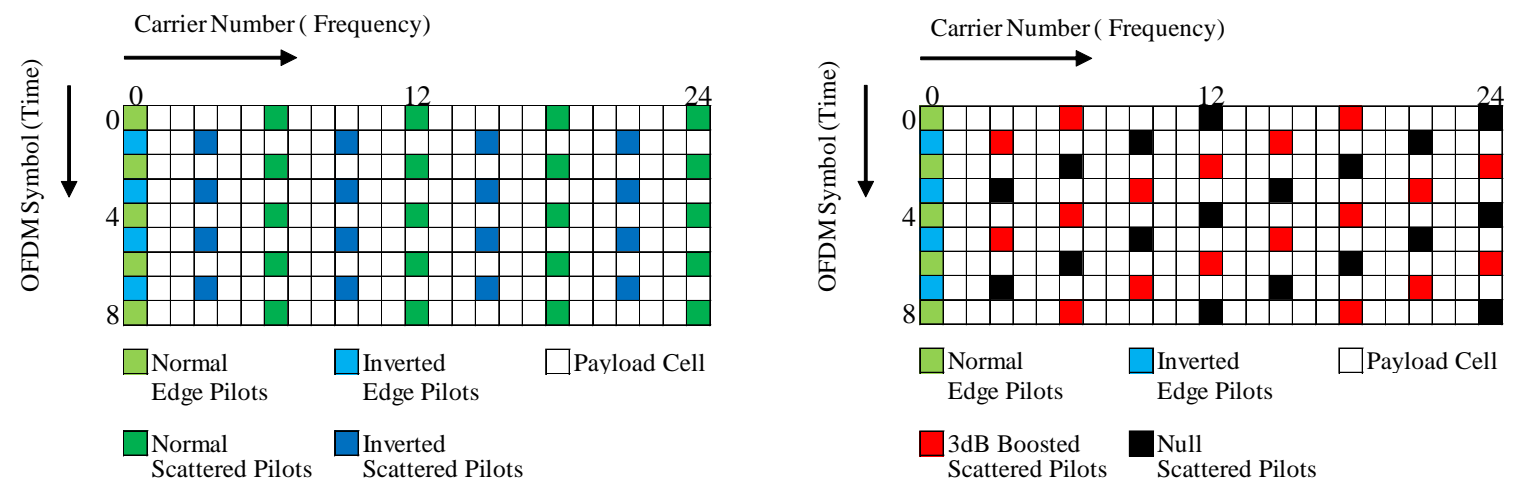

Fig. 1. MIMO pilots for Walsh-Hadamard encoding: MP3_2 (left), and Null pilot encoding: MP3_2 (right). Pilots for antenna \#2 are depicted for each encoding algorithm. Continual pilots are not shown.

\section{B. Null Pilot}

With Null pilot encoding, the amplitudes of the scattered pilots of both subsets are modified in both signals transmitted from antennas \#1 and \#2. With Null pilot encoding, antenna \#1 alternately transmits scattered pilots with $3 \mathrm{~dB}$ increased transmit power and scattered pilots with null power (zero amplitude). Scattered pilots of antenna \#2 are transmitted with null power and with $3 \mathrm{~dB}$ gain in reverse order. The $3 \mathrm{~dB}$ boosting keeps the total signal power of the scattered pilot to be the same as SISO. As the result of the nulling for the scattered pilot, the Nyquist limit of channel estimation in time equivalently falls to half compared to SISO. Additionally, the 3 $\mathrm{dB}$ boosting provides higher signal-to-noise-ratio (SNR) in the channel estimation results.

Taking into account a 2-dimentional channel interpolation (time interpolation followed by the frequency interpolation), the equivalent values of $D_{\mathrm{X}}$ and $D_{\mathrm{Y}}$ after channel interpolation in both MIMO pilot encoding algorithms are summarized in Table I.

TABLE I. $\quad$ EQUIVALENT $D_{\mathrm{X}}$ AND $D_{\mathrm{Y}}$ IN MIMO PILOTS

\begin{tabular}{c|c|c}
\hline \hline \multirow{2}{*}{ SISO } & \multicolumn{2}{|c}{ MIMO } \\
\cline { 2 - 3 } & $\begin{array}{c}\text { Walsh-Hadamard } \\
\text { encoding }\end{array}$ & Null pilot encoding \\
\hline \hline$D_{\mathrm{X}}$ & $2 D_{\mathrm{X}}$ & $D_{\mathrm{X}}$ \\
\hline$D_{\mathrm{Y}}$ & $D_{\mathrm{Y}}$ & $2 D_{\mathrm{Y}}$ \\
\hline \hline
\end{tabular}

\section{Evaluation Methodology}

The performance of MIMO scattered pilot is evaluated by physical layer simulations. The transmitter complies with the ATSC 3.0 specification. MIMO channel estimation algorithms have been implemented at the receiver side. The ideal noise estimation is adopted.

The first channel estimation step is to estimate the Channel Frequency Responses (CFRs) at the scattered pilot positions. The Least Square (LS) estimation is applied, which does not exploit the correlation of the channel across frequency and time [7]. The next step is channel interpolation. In order to reduce the complexity, channel interpolation is performed with a cascade of two 1-dimentional operations. First operation is a linear time interpolation to obtain CFRs at scattered pilot bearing carriers. Linear interpolation is a common option for time interpolation, since it only requires two points to be known. The second is frequency interpolation. Here, two common interpolations are investigated. One option is linear interpolation which is the computationally least expensive, but provides poor interpolation in cases where the data to be interpolated is non-linear. The second option is Discrete Fourier Transform (DFT) interpolation [8], [9]. The frequency interpolation is applied to fulfill the CFRs for all data carriers in a single OFDM symbol.

The two MIMO pilot encoding algorithms are compared in terms of Bit Error Rate (BER) after BCH. Minimum Square Error (MSE) between the estimated channel and the real channel is also evaluated. MSE is defined as:

$$
M S E=E_{l, k}\left[\frac{1}{4} \sum_{j=1}^{2} \sum_{i=1}^{2}\left\|h_{i j}[l, k]-\hat{h}_{i j}[l, k]\right\|^{2}\right]
$$

where $h_{i j}[l, k]$ and $\hat{h}_{i j}[l, k]$ denote the real and estimated CFR of receiving antenna $\# i$ and transmitting antenna \#j for carrier $k$ of the OFDM symbol number $l$, respectively. $E[\cdot]$ refers to the expectation calculation.

Both mobile and fixed reception scenarios have been considered with the NGH mobile outdoor channel [10] and a simple two path Single Frequency Network (SFN) channel. The SFN channel models a fixed receiver located between two MIMO transmitters using two configuration parameters: the power imbalance (PI), which gives the difference between the received signal powers from the two transmitters and the delay time between the received signals. In the following section, the PI $=3 \mathrm{~dB}$, the delay time $\tau=0.5$ GI duration are used for a typical fixed reception for SFN environment. The frequency offset between the two transmitters is set to $0 \mathrm{~Hz}$.

The transmission parameters for simulations are shown in Table II. In the simulation parameters, FFT size, modulation and GI ratio are selected to be the same as the operational parameters in current DTT system ISDB-T in Japan [11]. The required SNR of the current service for SISO rooftop reception is about $20 \mathrm{~dB}$. Using 64NUC 12/15, the SNR for MIMO would be about the same threshold with the same total transmitting power as SISO (i.e. half power in each antenna). The mobile reception service known as 1seg has been provided with QPSK 2/3 with SISO, and the QPSK 5/15 is selected as the parameters 
to provide the same capacity with MIMO. It is assumed that the cross polarization discrimination (XPD) is infinite as an ideal reception case. The performance is evaluated with a minimum mean-square error equalizer [12]. The densest pilot pattern MP3_2 is evaluated with long LDPC codes (64k) and nonuniform constellations standardized in ATSC 3.0 [13]. Note that SNR is defined as the ratio of the total transmitting signal power (antenna \#1 and \#2) to the noise power at each receiver (either antenna \#1 or \#2) in the following part.

TABLE II. TRANSMISSION PARAMETERS FOR SIMULATIONS

\begin{tabular}{l|c}
\hline \hline FFT size & $8 \mathrm{k}$ \\
\hline Number of carriers & 6913 \\
\hline Signal bandwidth & $5.83 \mathrm{MHz}$ \\
\hline Modulation and code rate & QPSK 5/15 \\
& 64 NUC 12/15 \\
\hline GI pattern & GI5_1024 \\
\hline Pilot pattern & MP3_2 $\left(D_{\mathrm{X}}=3, D_{\mathrm{Y}}=2\right)$ \\
\hline Pilot boosting & boost0 $\left(A_{\mathrm{sp}}=1.0\right)$ \\
\hline $\begin{array}{l}\text { MIMO scattered pilot } \\
\text { encoding }\end{array}$ & Whalsh-Hadamard encoding \\
\hline Frequency Interpolation & Null pilot encoding \\
\hline \hline
\end{tabular}

\section{EVALUATION RESULtS}

\section{A. NGH Mobile Outdoor Channel}

Fig. 2 shows MSE comparison for different MIMO pilot encoding in NGH outdoor channel $\left(F_{d}=33.3 \mathrm{~Hz}\right)$. This result shows that MSE of all configurations converges on a value around $-25 \mathrm{~dB}$ that is the tracking error for the time varying channel. Note that the MSEs in Fig. 2 become straight lines in the low SNR region (below $10 \mathrm{~dB}$ ), because the SNR is dominant compared with the tracking error. In the SNR region lower than $10 \mathrm{~dB}$, NP encoding shows lower MSE than WH because of the $3 \mathrm{~dB}$ boosting. It is assumed that the tracking error is greatly smaller than the AWGN at the receiver in lower SNR region. However, MSE caused by the tracking error can be observed in higher SNR region. In higher SNR region, NP encoding shows higher MSE than WH, because the virtually doubled equivalent $D_{\mathrm{Y}}$ provides poorer performance in time varying channel.

Fig. 3 shows BER performance of QPSK 5/15 and 64NUC $12 / 15$ in this channel. The result shows coherent results as MSE evaluation, i.e. the lowest MSE is obtained with NP-Linear and WH-Linear at around SNR $=6 \mathrm{~dB}$ and $28 \mathrm{~dB}$, respectively. We conclude that NP encoding is better in lower SNR region, but WH is better in higher SNR region in the mobile channel.

Regarding the frequency interpolation scheme, linear interpolation shows better performance than DFT interpolation. The linear interpolation process can be considered as a low-pass filtering, thus the linear interpolation equivalently reduces the noise bandwidth. It is considered that the channel is composed of some short echoes (up to $8.1 \mu$ s delay in time), thus linear frequency interpolation works well. Linear interpolation shows better performance regardless of the modulation scheme or the code rate for all SNRs.
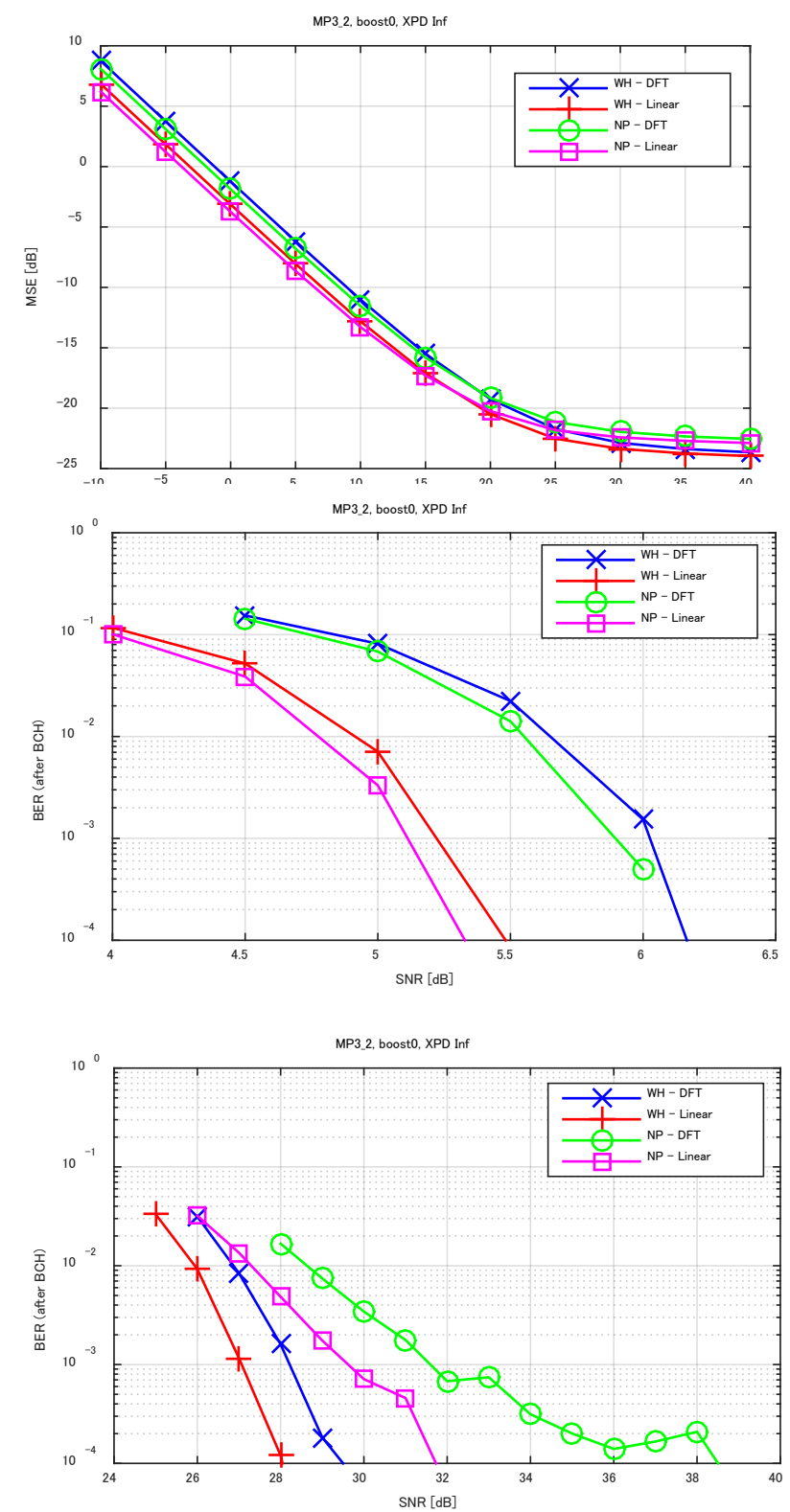

Fig. 3. Bit Error Rate comparison with Walsh-Hadamard and Null pilot encoding in NGH Mobile outdoor channel $\left(F_{d}=33.3 \mathrm{~Hz}\right)$ : QPSK5/15 (top), 64NUC 12/15 (bottom).

\section{B. Fixed SFN Channel}

Fig. 4 shows MSE comparison with both MIMO pilot encoding schemes in the SFN channel. This result shows that MSE of linear interpolation converges on a value that is the estimation error caused by the frequency interpolation. 


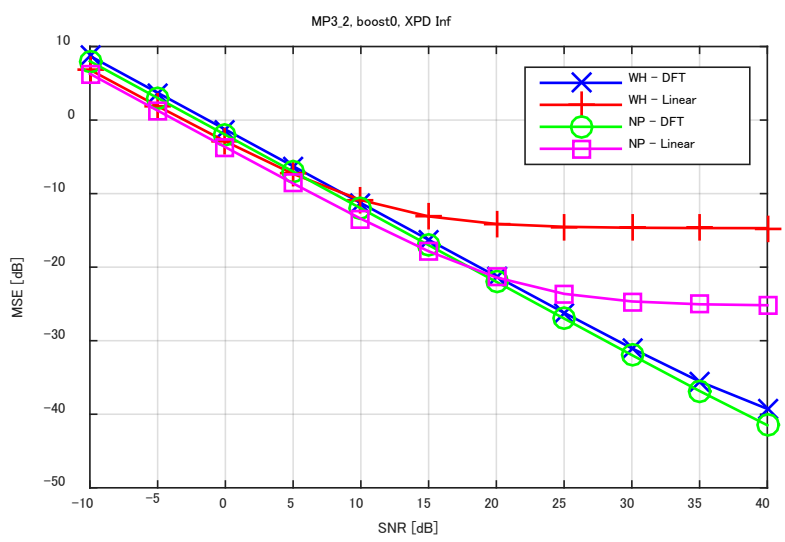

Fig. 4. MSE comparison of Walsh-Hadamard and Null pilot encoding in SFN channel (PI $=3 \mathrm{~dB}, \tau=0.5 \mathrm{GI})$.
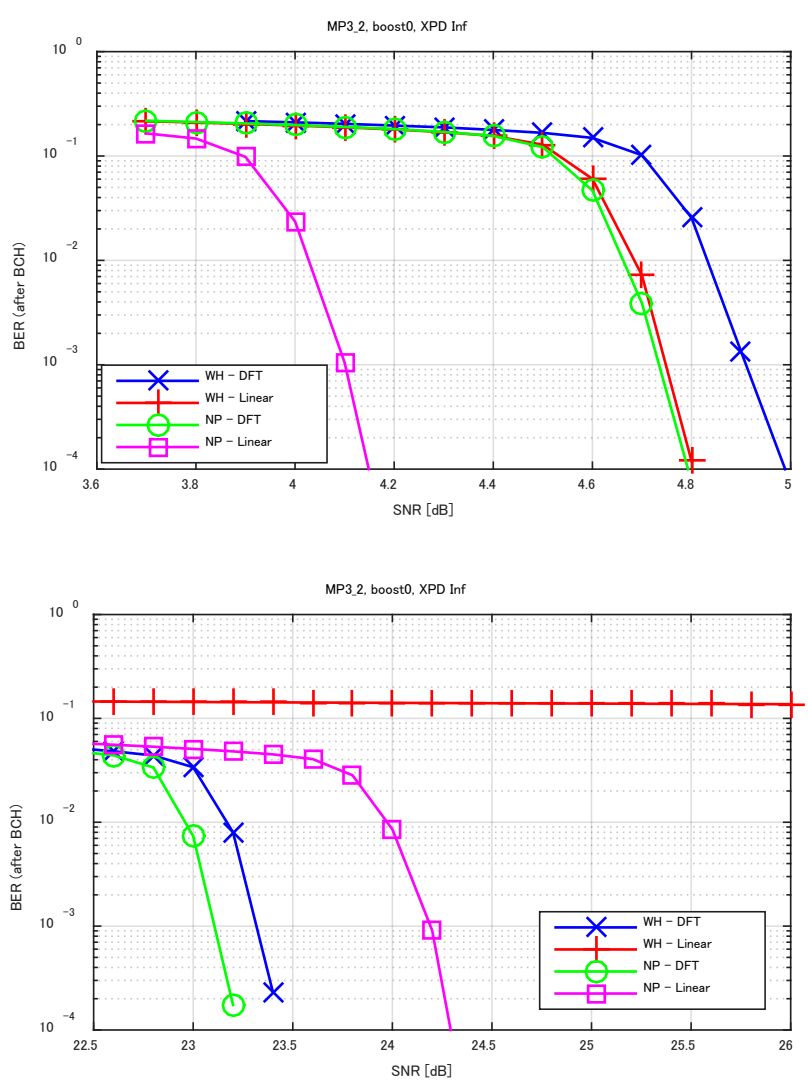

Fig. 5. Bit Error Rate comparison of Walsh-Hadamard and Null pilot encoding in SFN channel (PI $=3 \mathrm{~dB}, \tau=0.5 \mathrm{GI}$ ): QPSK 5/15 (top), 64NUC 12/15 (bottom).

Comparing WH and NP, NP shows lower MSE than WH in the SFN channel. The $3 \mathrm{~dB}$ boosting in NP encoding provides a lower MSE in such a static channel.

Fig. 5 shows BER performance of QPSK 5/15 and 64NUC $12 / 15$ in this channel. The lowest MSE is obtained with NPLinear and NP-DFT at around SNR $=4 \mathrm{~dB}$ and $23 \mathrm{~dB}$, respectively. We conclude that $\mathrm{NP}$ encoding is better in the fixed channel.

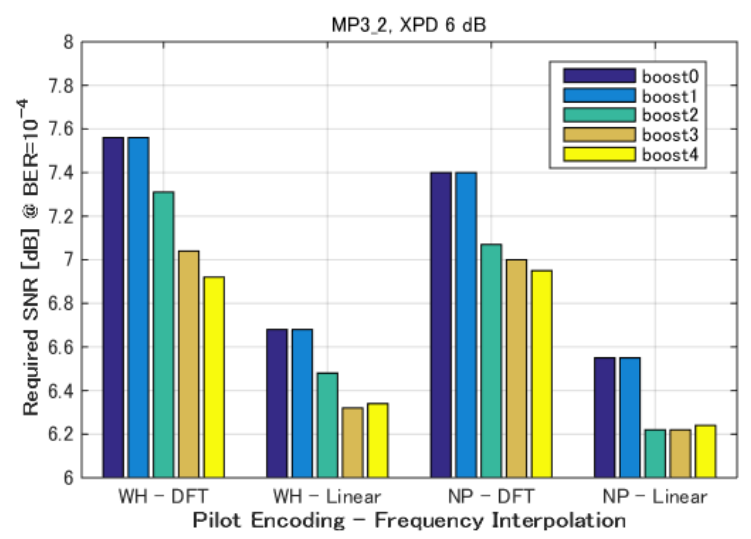

Fig. 6. Required SNR comparison for MIMO pilot encoding and frequency interpolation with MP3_2, QPSK 5/15 in NGH outdoor channel $\left(F_{d}=33.3 \mathrm{~Hz}\right)$.

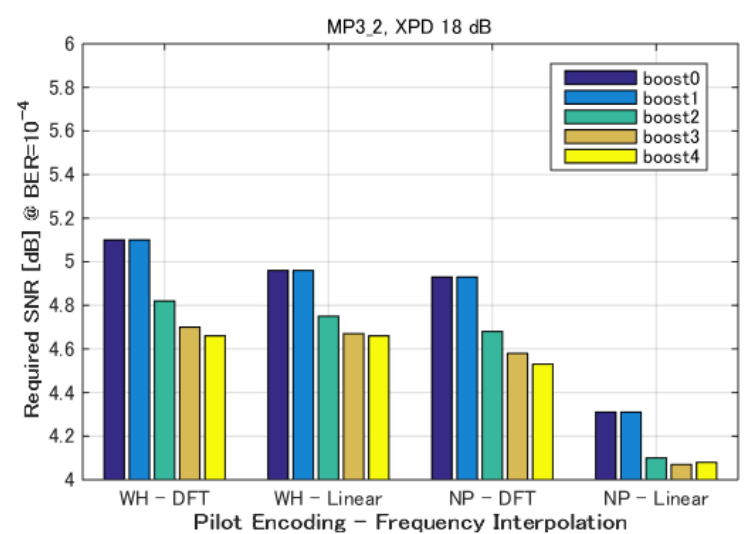

Fig. 7. Required SNR comparison for MIMO pilot encoding and frequency interpolation with MP3_2, QPSK 5/15 in SFN channel $(\mathrm{PI}=3 \mathrm{~dB}, \tau=0.5 \mathrm{GI})$.

Regarding the frequency interpolation, MSE with linear interpolation becomes much higher than DFT interpolation in higher SNR region, and it is assumed that the channel is not linear with such a long echo, thus the linear interpolation could not be able to interpolate the channel properly. In addition, it is confirmed that Quasi Error Free (QEF) is not achieved with 64NUC 12/15 WH-Linear configuration in the SFN channel, because the MSE is higher than the required SNR. On the other hand, the channel distortion can be properly interpolated with DFT interpolation, because the channel is static in time and the echo is within the Nyquist limit.

\section{Pilot Boosting}

We evaluated the effect of SP boosting with both pilot encoding algorithms and the frequency interpolation. The required SNR comparison for SP boosting with QPSK 5/15 in NGH outdoor channel is shown in Fig. 6. Here, XPD is set to 6 $\mathrm{dB}$ for a practical mobile reception scenario [10]. The result shows that the SP boosting can improve the required SNR for all SP configurations. It is confirmed that the best configuration is NP-Linear for each SP boosting value in the mobile channel.

Fig. 7 shows the results in the SFN channel. XPD is set to 18 $\mathrm{dB}$ as a practical fixed reception scenario [14]. The result shows 
that the SP boosting improves the required SNR and that the best configuration is NP-Linear for each SP boosting value. It is confirmed that the gain introduce by the pilot boosting varies depending on the channel, but the best configuration, i.e. the pilot encoding algorithm and the frequency interpolation, is not changed depending on the pilot boosting value.

\section{CONCLUSION AND FUTURE WORK}

This paper compares the two MIMO pilot encoding algorithms adopted in ATSC 3.0: Walsh-Hadamard and Null pilot encoding. The two pilot encoding schemes have been compared for different channel estimation algorithms (especially the interpolation method in frequency) for mobile and fixed channels using physical layer simulations. The results show that the encoding scheme and the frequency interpolation do not make much difference in the low SNR region. For SNRs below $10 \mathrm{~dB}$, Null pilot encoding and linear frequency interpolation is the best combination in mobile and fixed reception scenario. On the other hand, Walsh-Hadamard encoding can provide a better performance in mobile reception conditions especially at high SNRs. Linear frequency interpolation is not suited for SFNs with long echoes at high SNRs.

For future work, more pilot partners with different combinations of $D_{\mathrm{X}}$ and $D_{\mathrm{Y}}$ should be evaluated, together with the impact of the pilot boosting, to provide a complete MIMO pilot recommendation for ATSC 3.0.

\section{ACKNOWLEDGEMENT}

This work was partially supported by the Ministerio de Educacion y Ciencia, Spain (TEC2014-56483-R), co-funded by European FEDER funds.
[1] D. Gomez-Barquero, C. Douillard, P. Moss, and V. Mignone, "DVBNGH: The Next Generation of Digital Broadcast Services to Handheld Devices,” IEEE Trans. Broadcast., vol. 60, no. 2, pp. 246-257, June 2014.

[2] L. Fay, L. Michael, D. Gomez-Barquero, N. Ammar, and M. W. Caldwell, “An overview of the ATSC 3.0 physical layer specification," IEEE Trans. Broadcast., vol. 62, no. 1, pp. 159-171, Mar. 2016.

[3] D. Gomez-Barquero, et al., "MIMO for ATSC 3.0," IEEE Trans. Broadcast., vol. 62, no.1, pp. 298-305, Mar. 2016.

[4] S. Saito, et al., "8K Terrestrial Transmission Field Tests Using DualPolarized MIMO and Higher-Order Modulation OFDM," IEEE Trans. Broadcast., vol. 62, no.1, pp. 306-315, Mar. 2016.

[5] I. Eizmendi, et al, "DVB-T2: The Second Generation of Terrestrial Digital Video Broadcasting System,” IEEE Trans. Broadcast., vol. 60, no. 2, pp. 258-271, June 2014.

[6] E. Garro, J. Joan Gimenez, S.-I. Park, and D. Gomez-Barquero, “Scattered Pilot Performance and Optimization for ATSC 3.0," IEEE Trans. Broadcast., vol. 63, no. 1, pp. 282-291, Mar. 2017.

[7] M. K. Ozdemir and H. Arslan, "Channel estimation for wireless OFDM systems,” IEEE Commun. Surveys Tuts., vol. 9, no. 2, pp. 18-48, 2nd Quart., Apr. 2007.

[8] M. J. Fernandez-Getino Garcia, J. M. Paez-Borrallo, and S. Zazo, "DFTbased channel estimation in 2D Pilot Symbol Aided OFDM Wireless Systems,” Proc. IEEE VTC Spring 2001, Rhodes, Greece.

[9] A. Dowler, A. Doufexi, and A. Nix, "Performance evaluation of channel estimation techniques for a mobile fourth generation wide area OFDM systems,” Proc. IEEE VTC Fall 2002, Vancouver, Canada.

[10] P. Moss, T. Y. Poon, and J. Boyer, “A simple model of the UHF crosspolar terrestrial channel for DVB-NGH,” BBC Research \& Development White Paper WHP205, Sept. 2011.

[11] ITU-R Recommendation BT.1306-6, "Error-correction, Data Framing, Modulation and Emission Methods for Digital Terrestrial Television Broadcasting,” Nov. 2011.

[12] P. Fertl et al., "Performance Assessment of MIMO-BICM Demodulators Based on Mutual Information,” IEEE Trans. Signal Processing., vol. 60, no. 3, pp. 1366-1382, Mar. 2012.

[13] L. Michael and D. Gomez-Barquero, "Modulation and coding for ATSC3.0,” Proc. IEEE BMSB 2015, Ghent, Belgium.

[14] ITU-R Recommendation BT.419-3, "Directivity and polarization discrimination of antennas in the reception of television broadcasting," June 1990. 